\title{
A Simplified Design Inline Microstrip-to-Waveguide Transition
}

\author{
José M. Pérez-Escudero $^{1}$ (D), Alicia E. Torres-García ${ }^{1}$ (1) Ramón Gonzalo ${ }^{1,2}$ (1) \\ and Iñigo Ederra 1,2,* (iD) \\ 1 Antenna Group, Public University of Navarra, 31006 Pamplona, Spain; \\ josemanuel.perez@unavarra.es (J.M.P.-E.); aliciaelena.torres@unavarra.es (A.E.T.-G.); \\ ramon@unavarra.es (R.G.) \\ 2 Institute of Smart Cities, Public University of Navarra, 31006 Pamplona, Spain \\ * Correspondence: inigo.ederra@unavarra.es; Tel.: +34-948-16-60-45
}

Received: 31 August 2018; Accepted: 21 September 2018; Published: 23 September 2018 updates

\begin{abstract}
A simplified design of an inline transition between microstrip and rectangular waveguide is presented in this paper. The transition makes use of a dielectric filled rectangular waveguide (DFRW) as an intermediate step, which simplifies manufacturing and allows for an analytical design. The behavior of the transition has been experimentally validated in the W-band by means of a back-to-back configuration. Good performance has been achieved: a return loss greaterthan $10 \mathrm{~dB}$ and mean insertion loss lower than $1 \mathrm{~dB}$.
\end{abstract}

Keywords: waveguide transition; microstrip; Chebyshev transformer

\section{Introduction}

Over the last few years, an increasing number of applications at the millimeter and sub-millimeter ranges in different fields such as imaging [1], communications [2], radar [3], and automotive radar [4] has motivated research on the development of components and systems in this range. These have been facilitated by the availability of commercial monolithic microwave integrated circuits (MMICs) and the existence of low loss dielectric substrates. Among the components in development, those in planar technology [5,6] are becoming increasingly relevant. However, in these frequency ranges, the use of waveguide-based components and packages is still predominant. Therefore, transitions between planar transmission lines and waveguides have become essential for packaging and integration of these circuits with waveguide components, e.g. horn antennas. In addition, given the lack of connectors at submillimeter and terahertz frequencies, most test equipment is waveguide-based, which also makes these transitions required for component characterization. Since microstrip lines are widely used, this work concentrates on the design of a simple inline transition between this transition line and a rectangular waveguide that allows analytical design.

Different types for microstrip-to-waveguide transitions can be found in the literature. Among them, the E-plane probe is the most common. This transition is based on a microstrip line introduced through an aperture in the E-Plane of a rectangular waveguide that couples the quasi-TEM mode of the microstrip line to the $T E_{10}$ mode of the waveguide [7-10]. Some of these transitions have been presented operating in the full W-Band $[8,10]$, and feature insertion loss as low as $0.4 \mathrm{~dB}$, typically around $1 \mathrm{~dB}$. However, in order to avoid higher order mode excitation, they require a very narrow channel to introduce the microstrip probe. This problem can be alleviated by using Perfect Magnetic Conductor (PMC) lids [10], allowing for wide microstrip substrates, although at the expense of increased manufacturing complexity. Moreover, in E-plane probe transitions, the input port is usually perpendicular to the output port. This fact can make characterization of devices or antennas 
a cumbersome task, since additional bent sections must be added. Furthermore, the behavior of the transition is very sensitive to the position of the microstrip substrate with respect to the waveguide, in such a way that small errors during the fabrication process may produce large disagreements between simulation and experimental results. This problem repeats in other transitions based on radiating elements such as quasi-Yagi antennas [11] and slotline antennas [12].

On the other hand, the quasi-TEM microstrip mode can be easily coupled to the $T E_{10}$ rectangular waveguide mode by means of an inline configuration in which the field propagation direction in the waveguide is the same as in the microstrip line [13-19]. In the W-band, this concept was used in [19], where partially filled with dielectric waveguide matching sections were used. These sections were based on the gradual reduction of the dielectric material inside the waveguide. Therefore, they must be numerically optimized and, in addition, complicate manufacturing and assembly. A similar approach was followed in [20], where the band was extended at the expense of an increase of the number of sections. This length can be reduced by using a cavity excited by a microstrip probe [21], but the design requires full-wave optimization.

In this paper, an alternative design for the inline microstrip-to-waveguide transition is presented. The gradual transition between the dielectric filled waveguide and the air-filled section used in [19] is substituted by homogeneous waveguide sections. As a consequence, the proposed transition does not use any soldering and it does not require a ridge waveguide section or a gradual taper of the dielectric material, which can be difficult to manufacture for W-Band frequencies and above. It is, therefore, simpler than other inline transitions and easier to manufacture, which makes it appropriate for sub-millimeter and Terahertz frequencies.

In addition, the proposed configuration allows a simplified design based on standard impedance transformers. In this case, the transition will be designed by means of a Chebyshev adapter [22]. In addition, for the microstrip section, the authors first proposed a linear transition [23], which in this case is compared with a microstrip Chebyshev transformer. In both cases, an analytic calculation of the whole transition is proposed, which avoids full-wave optimization. Besides this comparison, the theoretical results in [23] are completed in this paper with the experimental verification of the concept.

The paper is organized as follows. The design procedure is presented in Section 2 and applied in Section 3 to the design of a W-band transition. Manufacturing of the transition is described in Section 4 and the experimental results are shown in Section 5. Finally, conclusions are drawn in Section 6.

\section{Configuration and Design Procedure}

The proposed transition first couples the quasi-TEM mode of the microstrip line to the $T E_{10}$ mode of a dielectric filled rectangular waveguide (DFRW). Afterwards, the transition from DFRW to standard rectangular waveguide is done by means of a multisection Chebyshev transformer. As a difference to previous designs, in this case, homogeneous waveguide sections will be used. This allows the design method to be completely analytic and make use of the Chebyshev transformer design procedure [22].

For the microstrip to DFRW section, two alternatives are proposed: a Chebyshev transformer (Figure 1a ) and a linear taper transition (Figure 1b). In both cases, the design of the two transition sections can be made analytically.

For the Chebyshev sections, the maximum value of the reflection coefficient can be calculated as

$$
A=\ln \left(\frac{Z_{L}}{Z_{D F R W}}\right) \frac{1}{2 T_{N}\left(\sec \left(\theta_{M}\right)\right)}
$$

where $N$ is the order of the Chebyshev transformer, $T_{N}$ is the Chebyshev polynomial of $N$ th order, $Z_{L}$ is the load impedance (standard waveguide), and $Z_{D F R W}$ is the input impedance (dielectric filled rectangular waveguide). This impedance and the impedance of the rectangular waveguide sections are computed by the equivalent electric waveguide impedance [24]: 


$$
Z_{w g}=2 \frac{\eta}{\sqrt{\epsilon_{r}}} \frac{b}{a}
$$

where $\eta=\sqrt{\frac{\mu_{0}}{\epsilon_{0}}}$ is the free-space wave-impedance, $\epsilon_{r}$ is the relative permittivity of the waveguide filling medium, $a$ is the waveguide width, and $b$ is the height of the waveguide sections.

The parameter $\theta_{M}$ is given by

$$
\theta_{M}=\frac{\pi}{2}\left(1-\frac{\Delta f}{2 f}\right)
$$

where $\Delta_{f}$ is the bandwidth of the transition, and $f$ is the central frequency.

Once this value is determined, the impedance of the different sections can be obtained by the standard formulation.
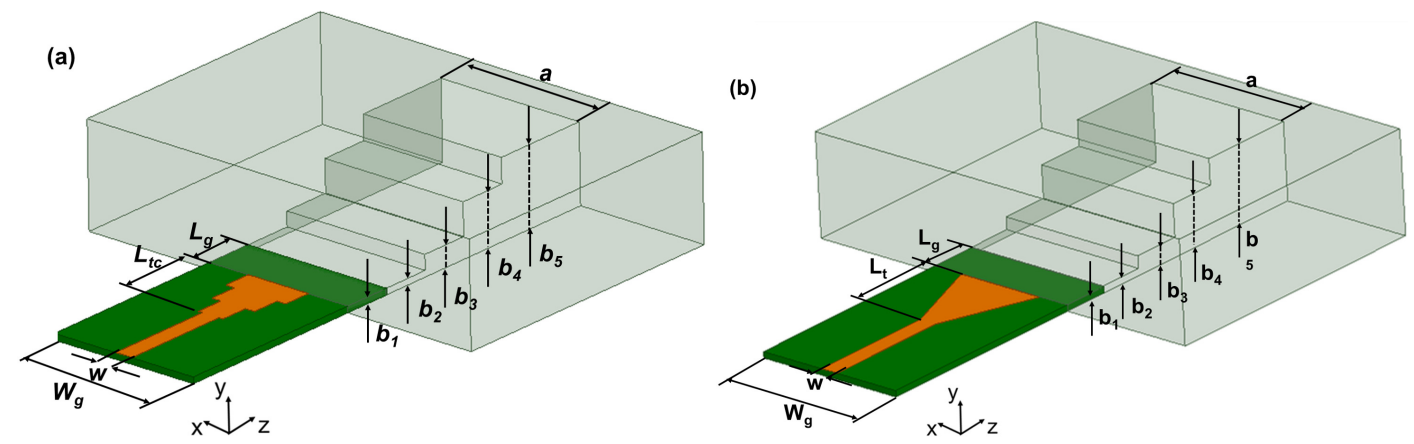

Figure 1. 3D geometry of the microstrip to standard rectangular waveguide (WR-10) transition: (a) microstrip Chebyshev transformer and (b) microstrip linear taper. The grey areas correspond to the rectangular waveguide metallic walls.

The Chebyshev transformer is a gradual method that matches a low impedance to a high impedance or vice versa. In this case, the DFRW impedance is an intermediate impedance lower than both the input microstrip line and the standard waveguide impedance. For instance, for the standard WR10 waveguide $a=2.54 \mathrm{~mm}$ and $b=1.27 \mathrm{~mm}$, leading to $Z_{L}=376.7 \Omega$, whereas for the dielectric filled rectangular waveguide $b$ corresponds to the substrate thickness. If a 0.1-mm-thick Rogers Cuclad $\left(\epsilon_{r}=2.4\right)$ is considered, $Z_{D F R W}=19 \Omega$. Therefore, the procedure for the calculation of the inline microstrip-to-rectangular-transition must be a two-step process. The first one is the design of the transition from DFRW to rectangular waveguide, presented in Figure 2. The second one involves the transition from microstrip to DFRW, shown in Figure 3a,b. The details of the design of both sections are given in the following sections.

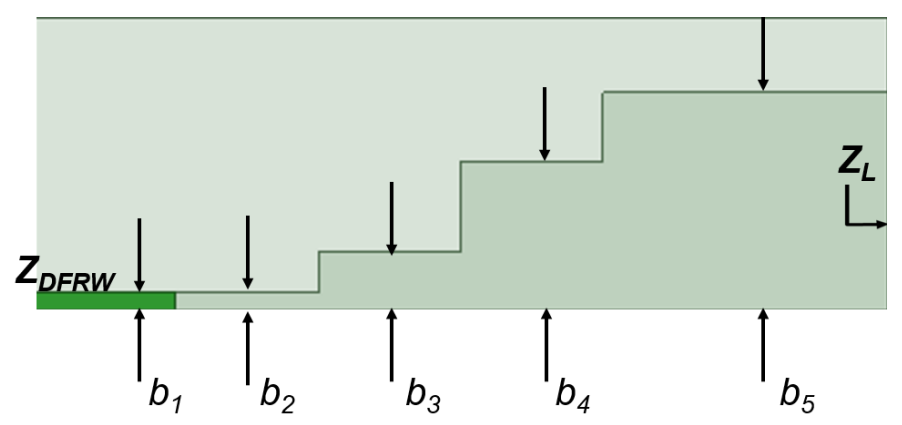

Figure 2. Side view of the multisection Chebyshev transformer from dielectric filled rectangular waveguide to standard rectangular waveguide. 
(a)

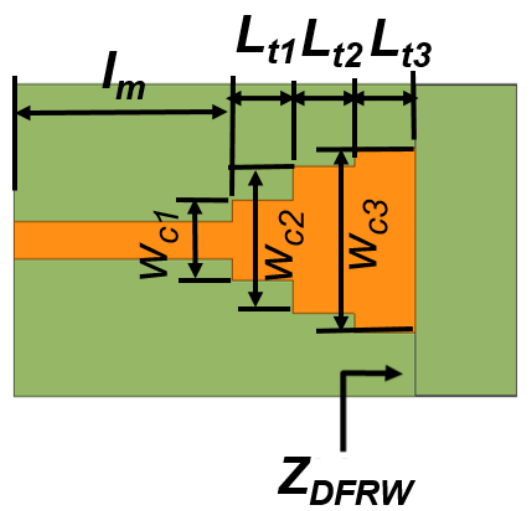

(b)

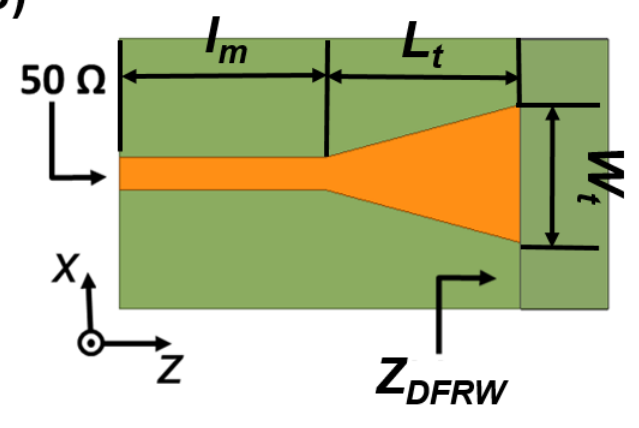

Figure 3. Top view of the microstrip to dielectric filled rectangular waveguide transition: (a) Chebyshev multisection transformer and (b) microstrip linear taper.

\subsection{Transition from Dielectric Filled Rectangular Waveguide to Standard Waveguide}

A multisection waveguide Chebyshev transformer is used to match the DFRW with the rectangular waveguide, see Figure 2. Once the DFRW and the standard rectangular waveguide impedances, $Z_{D F R W}$ and $Z_{L}$, have been obtained, we apply the Chebyshev transformer method to calculate the impedances of the matching sections.

With these impedance values, the height of each rectangular waveguide section is determined by

$$
b_{n}=\frac{Z_{w g_{n}} \sqrt{\epsilon_{r}} a}{2 \eta}
$$

where $Z_{w g_{n}}$ is the impedance of each waveguide section computed previously with the Chebyshev method.

Note that the first section corresponds to a dielectric filled waveguide, whereas the rest of them are air-filled waveguide sections. A more accurate transition could be designed taking into account the reactance effect due to the change of the waveguide height [25]. This effect should make the transformer sections shorter, however, if the change of height is small the effect may be negligible. In this design it has not been taken into account for simplicity since the difference is smaller than the tolerances of the manufacturing method. The three sections of the transition have a length $l_{g}$ equal to $\lambda_{g} / 4$ (at the central frequency).

It is noteworthy that the design is fully analytic and there is no need to optimize the transition parameters, which is a great advantage from the point of view of the computational time.

\subsection{Transition from Microstrip to Dielectric Filled Rectangular Waveguide}

The microstrip to DFRW transition can be carried out with two different approaches. The first one uses also a multisection Chebyshev impedance transformer. The second approach consists of a linear taper that matches the impedance of the microstrip line with the impedance of the DFRW.

\subsubsection{Multisection Microstrip Chebyshev Impedance Transformer}

The microstrip line is built on a substrate with thickness $\left(b_{1}\right)$ which corresponds to the height of DFRW. Taking this into account, Equation (2) is used to calculate the impedance of the DFRW, and Chebyshev transformer standard equations [22] are employed to determine the impedances of the transformer between $50 \Omega$ and this impedance. The width of the microstrip line sections are given by the well known formulae of the microstrip impedance [26]. The length of each section is $\lambda_{\text {eff }} / 4$ where $\lambda_{e f f}$ is the wavelength at the central frequency in each microstrip line section. 


\subsubsection{Tapered Microstrip}

An alternative way of matching the $50 \Omega$ impedance with the impedance of the DFRW can be seen in Figure $3 b$. This transition consists of a linear microstrip taper of length $L_{t}=3 \lambda_{\text {eff }} / 4$ (where $\lambda_{\text {eff }}$ is the guided wavelength of the microstrip line), which is enough to ensure good matching [27]. The width $W_{t}$ is calculated so that the characteristic impedance of the equivalent microstrip line is equal to the impedance of the DFRW.

\section{Design and Simulation Results}

The procedure above has been applied to the design of a microstrip to a $\mathrm{W}$-band rectangular waveguide inline transition. The microstrip line is printed on a 0.10-mm-thick Rogers Cuclad $\left(\epsilon_{r}=2.4\right.$ and $\tan \delta=0.009$ ). Losses in the copper line are also included in the simulations. The height of the dielectric filled rectangular waveguide (DFRW) $\left(b_{1}\right)$ is the thickness of the substrate, i.e., $0.1 \mathrm{~mm}$, and the width $(a)$ is the width of the standard WR-10 waveguide, i.e., $2.54 \mathrm{~mm}$.

The dimensions of the DFRW to WR-10 Chebyshev transformer were calculated with the previous procedure. To cover the full $\mathrm{W}$-band $\Delta f=35 \mathrm{GHz}$ (which leads to $\theta_{M}=1.2736$ ) is required. The maximum reflection coefficient is set to $-15 \mathrm{~dB}$, i.e., $A=0.032$. Three sections are required to comply with this requirement. Table 1 shows the dimensions of the required waveguide sections for the Chebyshev transformer. The labels are defined in Figure 2.

For the microstrip to DFRW section, both versions, i.e., the Chebyshev transition and linear taper, were designed. The Chebyshev transformer-based transition (see Figure 3a) consists of three sections, whose impedances were calculated with the same procedure above. Their dimensions are compiled in Table 2. For the linear taper (Figure $3 b$ ), the length $\left(L_{t}\right)$ was set to $3 \lambda_{\text {eff }} / 4$. The other dimensions are gathered in Table 3. For both configurations, full wave simulations have been carried out with Ansys HFSS from Ansys Electromagnetic Suite 17.1.

Table 1. Dimensions of the Chebyshev transformer waveguide sections $(\mu \mathrm{m})$.

\begin{tabular}{ccc}
\hline Parameter & Value & Fabricated Value \\
\hline$b_{1}$ & 100 & 100 \\
$b_{2}$ & 120 & 115 \\
$b_{3}$ & 320 & 347 \\
$b_{4}$ & 850 & 870 \\
$b_{5}$ & 1270 & 1270 \\
$l_{g 1}$ & 835 & 820 \\
$l_{g 2}$ & 835 & 817 \\
$l_{g 3}$ & 835 & 860 \\
\hline
\end{tabular}

Table 2. Dimension of the microstrip to DFRW Chebyshev transition $(\mu \mathrm{m})$.

\begin{tabular}{ccc}
\hline Parameter & Description & Value \\
\hline$a$ & Width of the rectangular waveguide & 2540 \\
$W$ & Width of the microstrip & 301 \\
$W_{c 1}$ & Width of section 1 & 450 \\
$W_{c 2}$ & Width of section 2 & 780 \\
$W_{c 3}$ & Width of section 3 & 1100 \\
$L_{t 1}$ & Length of section 1 & 493 \\
$L_{t 2}$ & Length of section 2 & 485 \\
$L_{t 3}$ & Length of section 3 & 481 \\
$L_{g}$ & Length of the DFRW & 830 \\
$l_{m}$ & Length of the microstrip & 2590 \\
\hline
\end{tabular}


Table 3. Dimension of the microstrip to DFRW linear taper transition $(\mu \mathrm{m})$.

\begin{tabular}{ccc}
\hline Parameter & Description & Value \\
\hline$L_{t}$ & Length of the taper & 1500 \\
$W_{t}$ & Width of the taper & 1300 \\
$l_{m}$ & Length of the microstrip & 2590 \\
\hline
\end{tabular}

The performance of the microstrip to DFRW transitions (both taper and Chebyshev line) and of the DFRW to rectangular waveguide Chebyshev transformer have been evaluated separately. The simulation results are shown in Figure 4. Return loss results are shown in Figure 4a, where we can appreciate that the linear taper microstrip line performs better than the Chebyshev transformer microstrip transition, both in return loss and insertion loss. For the DFRW to waveguide transition, the maximum return loss is in good agreement with the objective performance, a return loss higher than $15 \mathrm{~dB}$. This result justifies that the previously mentioned additional reactance created in the waveguide steps does not affect the transition performance. In Figure $4 \mathrm{~b}$, the $S_{21}$ parameter is presented. For all cases, the insertion losses are below $0.5 \mathrm{~dB}$.
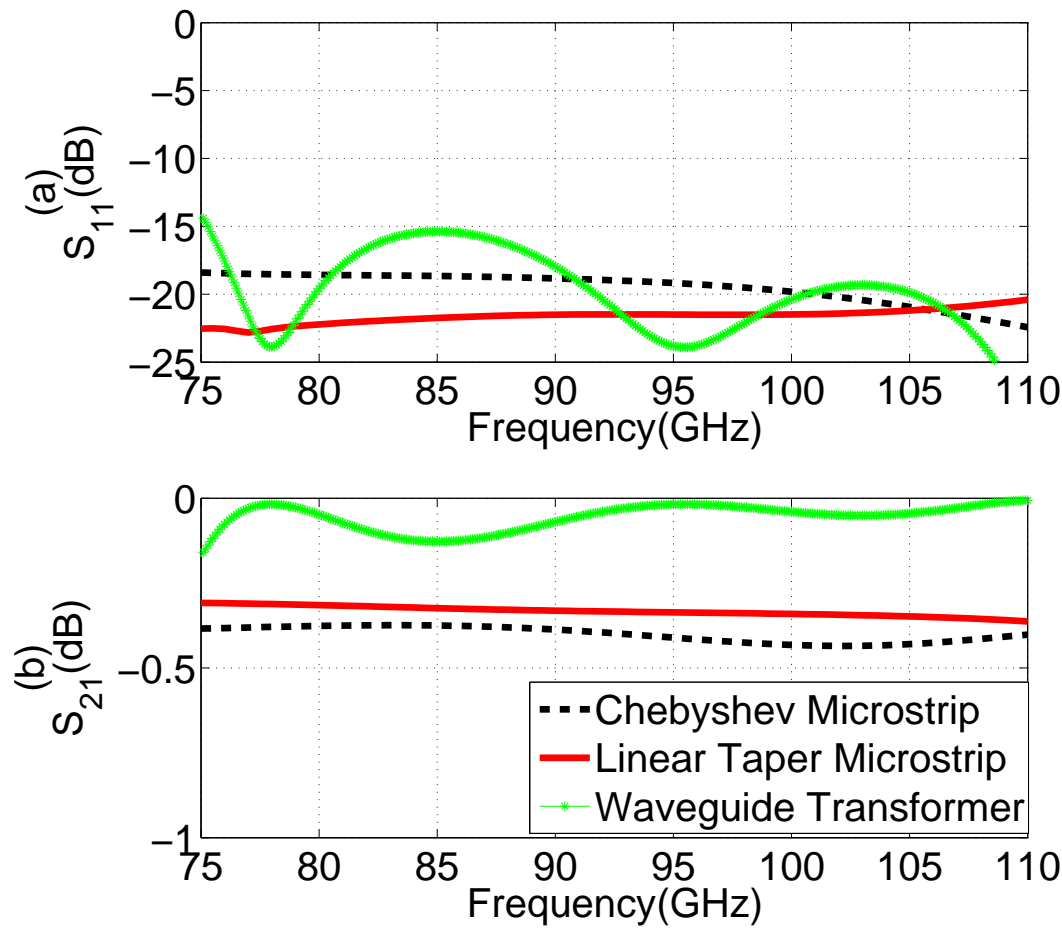

Figure 4. Performance evaluation of the different parts of the transition: (Solid red line) Linear microstrip to DFRW transition, (Dashed black line) Chebyshev microstrip to DFRW transition and (Green Star line) DFRW to rectangular waveguide Chebyshev transformer. (a) Reflection $\left(S_{11}\right)$; (b) Transmission $\left(S_{21}\right)$.

The predicted responses of the whole transitions are presented in Figure 5, where a comparison of the return loss between the linear taper and Chebyshev transitions is presented. It can be observed that the reflection $\left(S_{11}\right)$ is below $-15 \mathrm{~dB}$ in both cases. On the other hand, Figure $5 \mathrm{~b}$ shows the insertion loss. For both cases, the insertion losses are lower than $1 \mathrm{~dB}$ in the full W-band. Nevertheless, the Chebyshev case has larger insertion losses than those of the tapered transition. These losses are ascribed to the radiation in the steps between the different impedance sections of the Chebyshev transformer. Note that the transitions include a $2.59 \mathrm{~mm}$ microstrip line. The contribution of this line to the insertion losses above has been estimated as $0.33 \mathrm{~dB}$. If these losses are removed, the insertion loss of each transition can be estimated to be better than $0.5 \mathrm{~dB}$ and $0.8 \mathrm{~dB}$ for the linear and Chebyshev cases, respectively. 
Finally, it is worth pointing out that all these results have been accomplished with the dimensions resulting from the analytic procedure above. There has not been any optimization, and the objective return loss and bandwidth are fulfilled.
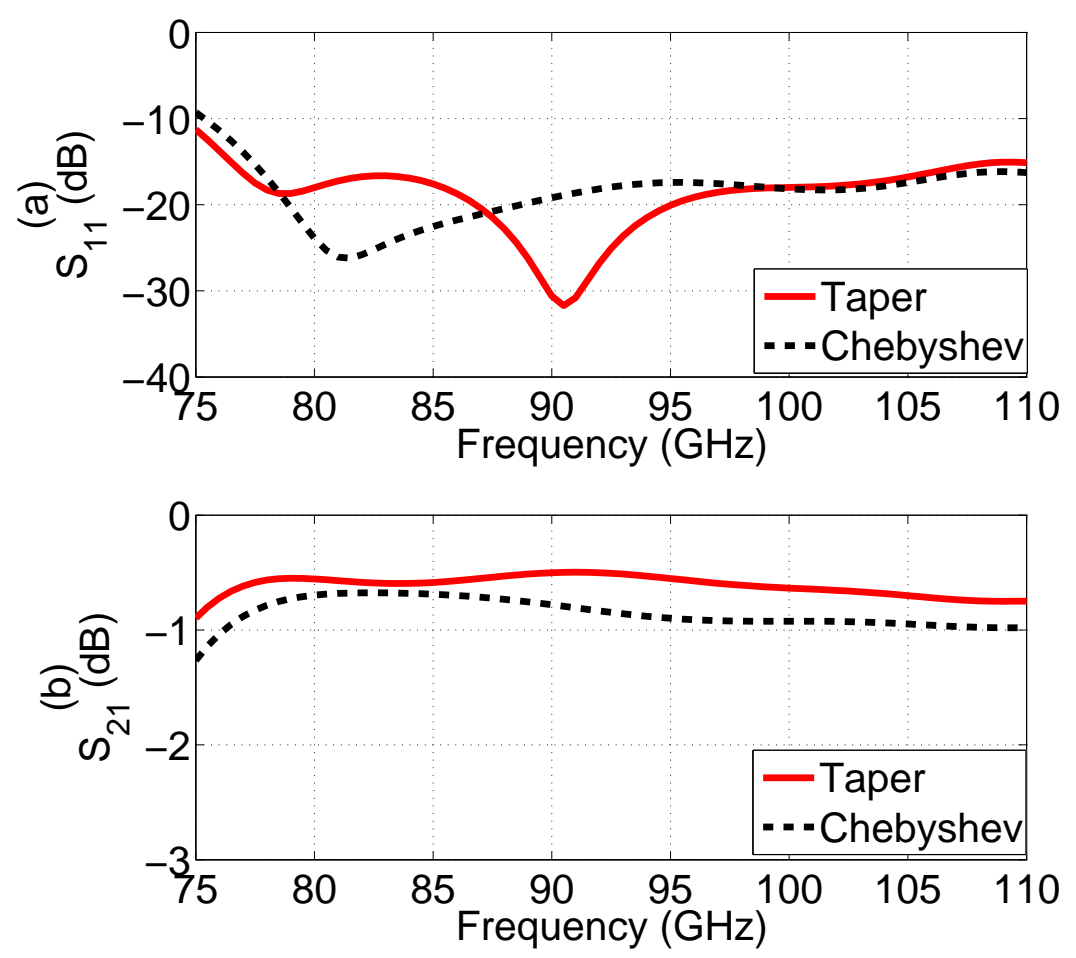

Figure 5. The simulation results of the two proposed whole transitions: (Dashed line) Chebyshev impedance transformer and (Solid line) microstrip taper. (a) Reflection $\left(S_{11}\right)$; and (b) Transmission $\left(S_{21}\right)$.

\section{Manufacturing}

Since the experimental validation will be carried out with a waveguide-based vector network analyzer, a back-to-back transition is needed. The two transitions will be connected by a $5.18 \mathrm{~mm}$ long microstrip line.

The manufactured process has to be separated in two parts: the milling process for the waveguide sections and the photolithographic process for the microstrip. Figure 6a shows the micromachined back-to-back waveguide blocks for the transition. They are made of aluminum by a high precision milling machine. To simplify assembly the waveguide block has been split in the H-plane, and both parts are screwed together. To reduce losses in the H-plane cut, this contact must be very tight, which is guaranteed by eight screws and four dowel pins. The block has been designed and manufactured with the appropriate WR-10 flange. The whole waveguide block is $34.2 \mathrm{~mm}$ long and $19.05 \mathrm{~mm}$ wide. The fabricated dimensions, shown in Table 1, are slightly different from the original design. The largest deviation correspond to the height of Section 3, where the error is $8 \%$. Note also that $0.150 \mathrm{~mm}$ radius rounded corners were created by the manufacturing method. The microstrip section was manufactured at the Public University of Navarra facilities following a standard photolithography procedure. The lines were created by sputtering the Cuclad substrate with 2 microns of $\mathrm{Cu}$. Finally, the substrate was cut with the required shape with a milling machine. Due to the better performance of the linear taper microstrip transition shown in simulation, only this one was fabricated and measured. The final circuit is shown in Figure $6 \mathrm{~b}$. 
(a)

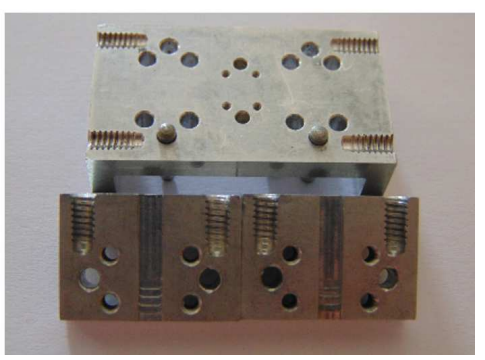

(b)

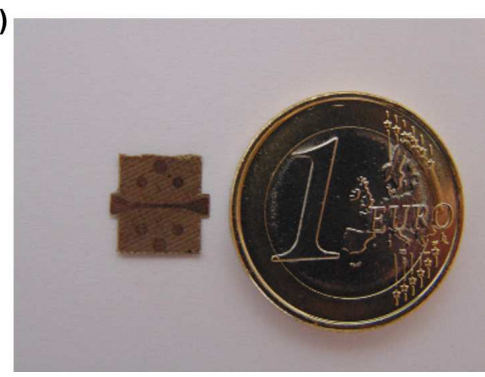

Figure 6. Photograph of (a) a fabricated back-to-back waveguide block; (b) a fabricated microstrip taper in Rogers Cuclad.

\section{Experimental Validation}

The set-up for measurements is shown in Figure 7. An Agilent PNA-X E3861 Microwave Network Analyzer with two VDI W-Band VNA Extenders was used. The equipment has been calibrated using the standard W-Band TRL (Through-Reflect-Line) calibration kit for VDI extenders. The manufactured back-to-back transition was connected in between the extender waveguide ports, and S parameter measurements were carried out.

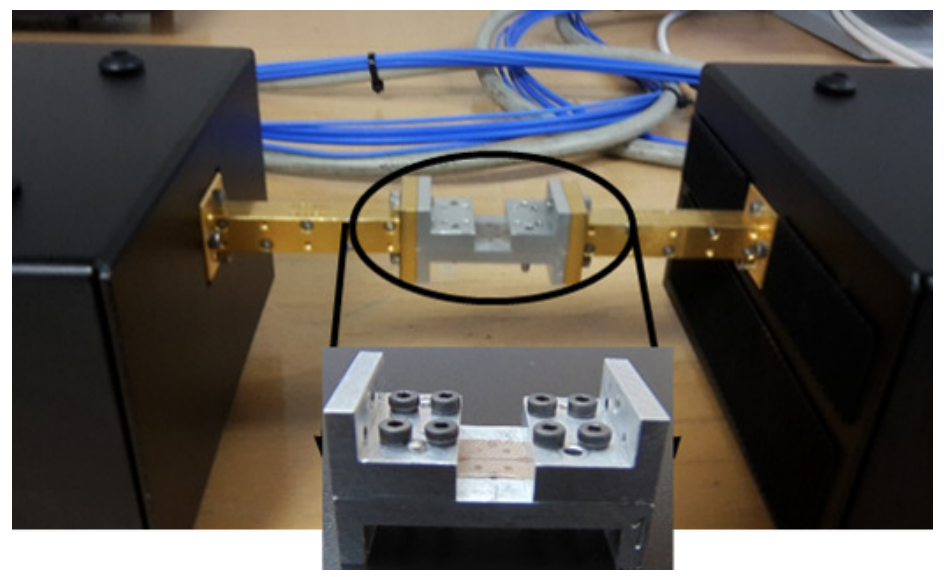

Figure 7. Setup for the experimental validation of the microstrip-to-waveguide transition.

The measured reflection coefficient, presented in Figure 8, shows a degradation of the performance with respect to the design, Figure 5. First, the working band has been shifted towards higher frequencies. For this reason, the return loss between 75 and $80 \mathrm{GHz}$ becomes lower than $10 \mathrm{~dB}$. In addition, the return loss increases to nearly $12 \mathrm{~dB}$ in the central frequency. However, taking into account the whole band, they are higher than $8 \mathrm{~dB}$. The insertion losses also increase, and the measured values are between 0.4 and $0.7 \mathrm{~dB}$ higher than the predictions. The obtained average value is $1.9 \mathrm{~dB}$.

This degradation is ascribed to the manufacturing errors. These errors can be noticed in Table 1 . They are particularly large in section 3, with more than $20 \mu \mathrm{m}$ difference in both length and height. The real dimensions have been simulated in order to perform a fair comparison with the measurements. This comparison is shown in Figure 8. There is good agreement between them, especially from $75 \mathrm{GHz}$ to the central frequency, $92.5 \mathrm{GHz}$. The higher return loss was adequately predicted. The transmission coefficient is displayed in Figure $8 \mathrm{~b}$. The mean value of the simulated and measured insertion losses is $1.5 \mathrm{~dB}$ and $1.9 \mathrm{~dB}$, respectively. These losses include those in the transitions and in the $5.18 \mathrm{~mm}$ microstrip line. Taking into account the value of $\tan \delta=0.009$ and the conductivity of copper, this microstrip line has a $0.65 \mathrm{~dB}$ insertion loss. If these losses are removed, the average insertion loss of each transition can be assumed to be $0.63 \mathrm{~dB}$. 

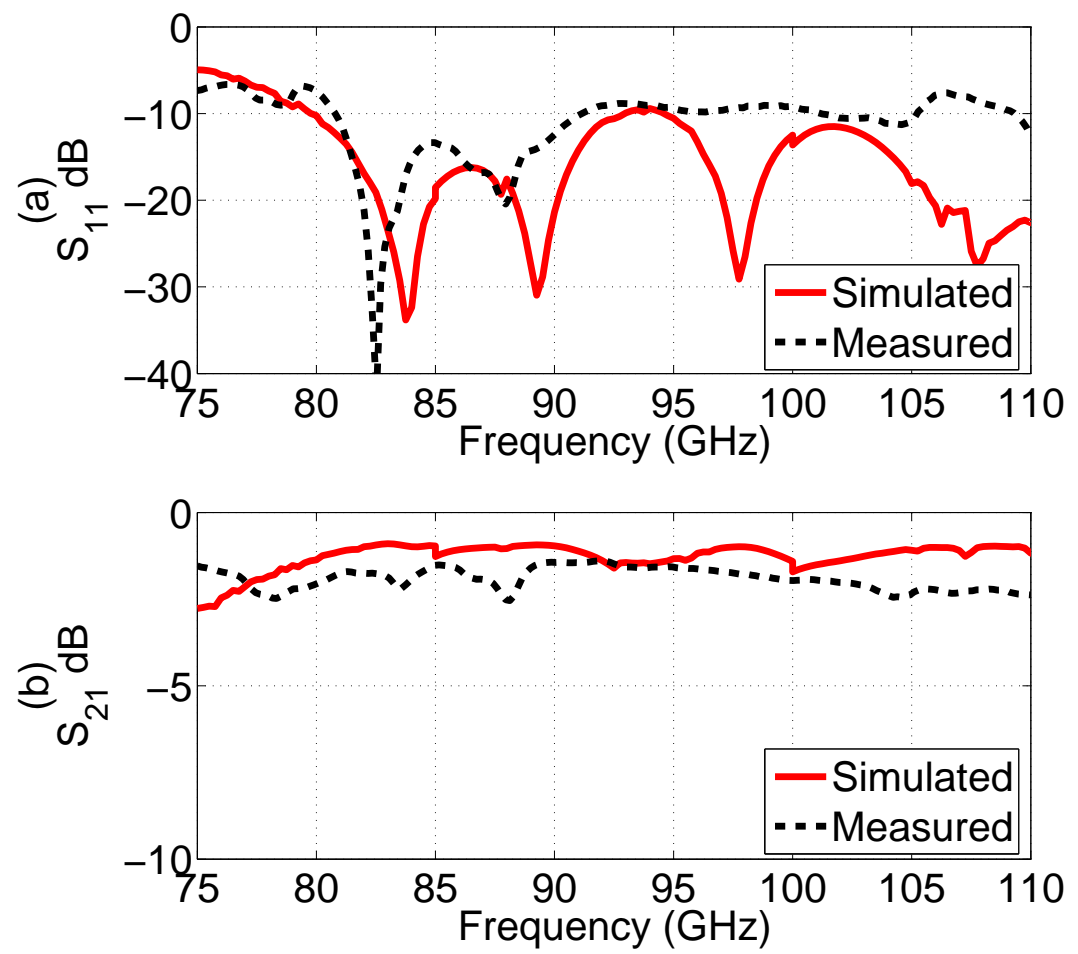

Figure 8. Simulated (solid line) and measured (dashed line) back-to-back transition. (a) Reflection $\left(S_{11}\right)$ and (b) Transmission $\left(S_{21}\right)$.

\section{Conclusions}

A simple inline microstrip-to-rectangular-waveguide transition, which allows for analytical design, has been presented in this paper. In addition, the configuration used in this work is simpler and easier to manufacture than other similar inline transitions. To validate the concept, a W-band transition has been designed. The simulation results show good performance for the whole W-Band: return losses are greater than $15 \mathrm{~dB}$, and insertion losses are below $1 \mathrm{~dB}$. The experimental validation, using a back-to-back transition, presents a slight degradation in performance, an $8 \mathrm{~dB}$ return loss and a $0.63 \mathrm{~dB}$ insertion loss, in good agreement with the predicted performance of the manufactured prototype.

Author Contributions: Investigation: J.M.P.-E., A.E.T.-G., R.G., and I.E.; writing—original draft: J.M.P.-E.; writing-review \& editing: I.E.

Funding: This research was funded by the Spanish Ministry of Economy and Competitiveness, grant number TEC2013-47753-C3-1-R and TEC2016-76997-C3-1-R.

Conflicts of Interest: The authors declare no conflict of interest.

\section{Abbreviations}

The following abbreviations are used in this manuscript:

MMIC monolithic microwave integrated circuits

PMC perfect magnetic conductor

DFRW dielectric filled rectangular waveguide

HFSS high frequency structure simulator 


\section{References}

1. Yujiri, L.; Shoucri, M.; Moffa, P. Passive Millimeter-Wave Imaging. IEEE Microw. Mag. 2003, 4, 39-50. [CrossRef]

2. Nagatsuma, T.; Ducournau, G.; Renaud, C.C. Advanced in terahertz communications accelerated by photonics. Nat. Photon. 2016, 10, 371-379. [CrossRef]

3. Golcuk, F.; Kanar, T.; Rebeiz, G.M. A 90-100 GHz 4x4 SiGe BiCMOS Polarimetric Transmit/Receive Phased Array with Simultaneous Receive-Beams Capabilities. IEEE Trans. Microw. Theory Tech. 2013, 61, 3099-3114. [CrossRef]

4. Folster, F.; Rohling, H.; Lubbert, U. An Automotive Radar Network Based on 77 GHz FMCW sensors. In Proceedings of the IEEE International Radar Conference, Arlington, VA, USA, 9-12 May 2005; pp. 871-876.

5. Lynch, J.J.; Moyer, H.P.; Schaffner, J.H.; Royter, Y.; Sokolich, M.; Hughes, B.; Yoon, Y.J.; Schulman, J.N. Passive Millimeter-Wave Imaging Module With Preamplified Zero-Bias Detection. IEEE Trans. Microw. Theory Tech. 2008, 56, 1592-1600. [CrossRef]

6. Rebollo, A.; Maestrojuan, I.; Gonzalo, R.; Ederra, I. A broadband radiometer configuration at $94 \mathrm{GHz}$ in planar technology. In Proceedings of the 2011 IEEE MTT-S International Microwave Workshop Series on Millimeter Wave Integration Technologies, Sitges, Spain, 15-16 September 2011; pp. 89-92.

7. Leong, Y.-C.; Weinreb, S. Full Band Waveguide-to-Microstrip Probe Transitions. In Proceedings of the 1999 IEEE MTT-S International Microwave Symposium Digest, Anaheim, CA, USA, 13-19 June 1999; Volume 4, pp. 1435-1438.

8. Shireen, R.; Shi, S.; Prather, D.W. W-Band Microstrip-to-Waveguide Transition Using Via Fences. Prog. Electromagn. Res. Lett. 2010, 16, 151-160. [CrossRef]

9. Li, E.S.; Tong, G.-X.; Niu, D.C. Full W-Band Waveguide-to-Microstrip Transition with New E-Plane Probe. IEEE Microw. Wirel. Compon. Lett. 2013, 23, 4-6. [CrossRef]

10. Zaman, A.U.; Vassilev, V.; Kildal, P.S.; Zirath, H. Millimeter Wave E-Plane Transition From Waveguide to Microstrip Line With Large Substrate Size Related to MMIC Integration. IEEE Microw. Wirel. Compon. Lett. 2016, 26, 481-484. [CrossRef]

11. Kaneda, N.; Qian, Y.; Itoh, T. A Broad-band Microstrip-to-Waveguide Transition Using quasi-Yagi Antenna. IEEE Trans. Microw. Theory Tech. 1999, 47, 2562-2567. [CrossRef]

12. Yang, M.H.; Xu, J.; Zhao, Q.; Li, G.P.; Peng, L. Compact, Broadband Waveguide-to-Microstrip Transition Using Slotline Antenna. In Proceedings of the 2010 International Conference on Microwave and Millimeter Wave Technology, Chengdu, China, 8-11 May 2010; pp. 744-747.

13. Yao, H.W.; Abdelmonem, A.; Liang, J.F.; Zaki, K.A. Analysis and Design of Microstrip-to-Waveguide Transitions. IEEE Trans. Microw. Theory Tech. 1994, 42, 2371-2380.

14. Boukari, B.; Moldovan, E.; Affes, S.; Wu, K.; Bosisio, R.G.; Tatu, S.O. Robust Microstrip-to-Waveguide Transitions for Millimeter-Wave Radar Sensor Applications. IEEE Antennas Wirel. Propag. Lett. 2009, 8, 759-762. [CrossRef]

15. Zhang, Y.; Ruiz-Cruz, J.A.; Zaki, K.A.; Piloto, A.J. A Waveguide to Microstrip Inline Transition with Very Simple Modular Assembly. IEEE Microw. Wirel. Compon. Lett. 2010, 20, 480-482. [CrossRef]

16. Simone, M.; Fanti, A.; Valente, G.; Montisci, G.; Ghiani, R.; Mazzarella, G. A Compact In-LineWaveguideto-Microstrip Transition in the Q-Band for Radio Astronomy Applications. Electronics 2018, 7, 24. [CrossRef]

17. Li, J.; Li, L.; Qiao, Y.; Chen, J.; Chen, J.; Zhang, A. Full Ka Band Waveguide-to-Microstrip Inline Transition Design. J. Infrared Millim. Terahertz Waves 2018, 39, 714-722. [CrossRef]

18. Scheiner, B.; Mann, S.; Lurz, F.; Michler, F.; Erhardt, S.; Lindner, S.; Weigel, R.; Koelpin, A. Microstrip-to-waveguide transition in planar form using a substrate integrated waveguide. In Proceedings of the 2018 IEEE Radio and Wireless Symposium (RWS), Anaheim, CA, USA, 15-18 January 2018; pp. 18-20.

19. Rebollo, A.; Gonzalo, R.; Ederra, I. An Inline Microstrip-to-Waveguide Transition Operating in the Full W-Band. J. Infrared Millim. Terahertz Waves 2015, 36, 734-744. [CrossRef]

20. Granja, A.B.; Jakoby, R.; Penirschke, A. Outright W-Band Chebyshev-based Hollow Waveguide to Microstrip Transition. In Proceedings of the 2017 42nd International Conference on Infrared, Millimeter, and Terahertz Waves (IRMMW-THz), Cancun, Mexico, 27 August-1 September 2017.

21. Zaman, A.U.; Vassilev, V.; Zirath, H.; Rorsman, N. Novel Low-Loss Millimeter-Wave Transition from Waveguide-to-Microstrip Line Suitable for MMIC Integration and Packaging. IEEE Microw. Wirel. Compon. Lett. 2017, 27, 1098-1100. [CrossRef] 
22. Orfanidis, S.J. Electromagnetic Waves and Antennas. 2016, pp. 614-638. Available online: http://www.ece. rutgers.edu/ orfanidi/ewa (accessed on 4 August 2017).

23. Pérez, J.M.; Rebollo, A.; Gonzalo, R.; Ederra, I. An Inline Microstrip-to-Waveguide Transition Operating in the Full W-Band Based on a Chebyshev Multisection Transformer. In Proceedings of the 2016 10th European Conference on Antennas and Propagation (EuCAP), Davos, Switzerland, 10-15 April 2016.

24. Eshrah, I.A.; Kishk, A.A.; Yakovlev, A.B.; Glisson, A.W. Rectangular Waveguide with Dielectric-Filled Corrugations Supporting Backward Waves. IEEE. Trans. Microw. Theory Tech. 2005, 53, 3298-3304. [CrossRef]

25. Marcuvitz, N. Waveguide Handbook; McGraw-Hill: New York, NY, USA, 1951; pp. 307-310.

26. Pozar, D.M. Microwave Engineering; John Wiley \& Sons: Hoboken, NJ, USA, 2011; pp. 256-261.

27. Deslandes, D. Design Equations for Tapered Microstrip-to-Substrate Integrated Waveguide Transitions. In Proceedings of the 2010 IEEE MTT-S International Microwave Symposium, Anaheim, CA, USA, 23-28 May 2010.

(C) 2018 by the authors. Licensee MDPI, Basel, Switzerland. This article is an open access article distributed under the terms and conditions of the Creative Commons Attribution (CC BY) license (http://creativecommons.org/licenses/by/4.0/). 